\title{
In vitro/in silico approach in the development of simvastatin-loaded self-microemulsifying drug delivery systems
}

\section{Zora Ćetković, Sandra Cvijić \& Dragana Vasiljević}

To cite this article: Zora Ćetković, Sandra Cvijić \& Dragana Vasiljević (2017): In vitro/in silico approach in the development of simvastatin-loaded self-microemulsifying drug delivery systems, Drug Development and Industrial Pharmacy, DOI: 10.1080/03639045.2017.1414835

To link to this article: https://doi.org/10.1080/03639045.2017.1414835

Accepted author version posted online: 12

Dec 2017.

Submit your article to this journal $\pi$

Џ Article views: 1

Q View related articles $\asymp$

View Crossmark data $\nearrow$ 
In vitro/in silico approach in the development of simvastatin-loaded selfmicroemulsifying drug delivery systems

Zora Ćetković $^{1}$, Sandra Cvijić ${ }^{1}$, Dragana Vasiljević ${ }^{1}$

${ }^{1}$ Department of Pharmaceutical Technology and Cosmetology, University of Belgrade Faculty of Pharmacy, Vojvode Stepe 450, 11221 Belgrade, Serbia

Corresponding author: Zora Ćetković, Department of Pharmaceutical Technology and Cosmetology, University of Belgrade - Faculty of Pharmacy, Vojvode Stepe 450, 11221 Belgrade, Serbia, tel: +381 11 3951366, e-mail: zora_cetkovic@yahoo.com

Zora Ćetković graduated from the University of Belgrade-Faculty of Pharmacy in 2010. During her studies, she received many scholarships and awards: scholarship of the City of Belgrade, scholarship from the budget of Republic of Serbia, scholarship of the Serbian Government, and the award for the extraordinary achievment in the field of pharmacy and medical biochemistry 'Professor Ivan Berkeš' (2010). She is a student of the doctoral academic studies at the University of Belgrade-Faculty of Pharmacy. From March 2012 to April 2014 she was an assistant at the Institute of Virology, Vaccines and Sera Torlak. From April 2014 to February 2015 she was a pharmacist at the Department of Compounding, Clinical Centre of Serbia. She currently holds the position of chief pharmacist at Clinic of Otorhinolaryngology and Maxillofacial Surgery, and also works as a hospital pharmacist at Emergency Centre, Clinical Centre of Serbia. 
Sandra Cvijić graduated from the University of Belgrade-Faculty of Pharmacy in 2003. In 2011, she defended her doctoral dissertation at the same faculty. During 2013, as a part of a DAAD Foundation scholarship, she attended postdoctoral training at the Department of Biopharmaceutical and Pharmaceutical Technology, Institute of Pharmacy and Biochemistry, Johannes Gutenberg University of Mainz. She is employed at the University of BelgradeFaculty of Pharmacy, as assistant professor. Since 2011 she has been engaged in the project of technological development 'Development of products and technologies that provide the desired release from solid dosage forms' financed by the Ministry of Education, Science and Technological Development of the Republic of Serbia, and from 2016 on the international project SimInhale COST Action MP1404. She is the author or co-author of a large number of press releases. She is also a reviewer for the following journals: Molecular Pharmaceutics; European Journal of Pharmaceutics and Biopharmaceutics; AAPS PharmSciTech; Drug Design, Development and Therapy; Asian Journal of Pharmaceutics.

Dragana Vasiljevic received her BSc (1986) at the University of Belgrade-Faculty of Pharmacy, Serbia. She completed MSc (1992) and PhD (2009) in Pharmaceutical technology at the University of Belgrade-Faculty of Pharmacy. She is employed at the University of Belgrade-Faculty of Pharmacy, as associate professor. Since 2011 she has been engaged in the project of technological development 'Development of products and technologies that provide the desired release from solid dosage forms' financed by the Ministry of Education, Science and Technological Development of the Republic of Serbia. Dragana Vasiljevic research interest is focused on formulation and characterization of emulsion systems and selfemulsifying drug delivery systems (SEDDS), semisolid preparations for cutaneous application, cosmetic products (emulsions and gels for various purposes), rheological 
characterization of dispersed systems. She is the author or the coauthor of more than 40 scientific papers and professional papers.

\section{Novelty statement}

The manuscript deals with the specific formulation approach (SMEDDS formulation) to improve systemic bioavailability of the poorly soluble model compound, and the employment of a comprehensive in vitro-in silico analysis to help identify the appropriate drug-specific formulation strategy. We believe that the results of this study may serve as a roadmap to define a proper formulation strategy for other poorly soluble drugs that undergo complex pharmacokinetics. 
In vitro/in silico approach in the development of simvastatin-loaded selfmicroemulsifying drug delivery systems

Objective: The aims of this study were to formulate simvastatin (SV)-loaded selfmicroemulsifying drug delivery systems (SMEDDS), and explore the potential of these drug delivery systems to improve SV solubility, and also to identify the optimal place in the gastrointestinal (GI) tract for the release of SV using coupled in vitro/in silico approach.

Significance: In comparison to other published results, this study considered the extensive presystemic clearance of SV, which could significantly decrease its systemic and hepatic bioavailability if SV is delivered into the small intestine. Methods: SV-loaded SMEDDS were formulated using various proportions of oils (PEG 300 oleic glycerides, propylene glycol monocaprylate, propylene glycol monolaurate), surfactant (PEG 400 caprylic/capric glycerides) and cosurfactant (polysorbate 80) and subjected to characterization, and physiologically-based pharmacokinetic (PBPK) modeling. Results: According to the in vitro results, the selected SMEDDS consisted of 10.0\% PEG 300 oleic glycerides, 67.5\% PEG 400 caprylic/capric glycerides and $22.5 \%$ polysorbate 80 . The use of acid-resistant capsules filled with SV-loaded SMEDDS was found helpful in protecting the drug against early degradation in proximal parts of the GI tract, however in silico simulations indicated that $\mathrm{pH}$-controlled drug release system that dissolve in distal parts of the intestine might further improve SV bioavailability (up to $7.20 \%$ ). Conclusions: The obtained results suggested that combined strategy for the improvement of SV bioavailability should comprise solubility enhancement and delayed drug release. The developed SV-specific PBPK model could potentially be used to assess the influence of formulation factors on drug absorption and disposition when developing SV oral dosage forms.

Keywords: self-microemulsifying drug delivery systems (SMEDDS), simvastatin, poor solubility, absorption simulation, physiologically-based pharmacokinetic modeling 


\section{Introduction}

Simvastatin (SV) is a cholesterol-lowering drug recognised for its potential to prevent cardiovascular diseases, since it treats hypercholesterolemia and can postpone the process of atherosclerosis [1]. SV is a lactone prodrug transformed into active form, $\beta, \delta$-dihydroxyacid by nonspecific carboxyesterases in the intestinal wall, liver, and to some extent in plasma or by nonenzymatic hydrolysis [2]. Simvastatin acid (SVA) is responsible for lowering cholesterol levels through reversible and competitive inhibition of 3-hydroxy-3methylglytaril coenzyme A reductase, an enzyme involved in the biosynthesis of cholesterol in the liver [1]. In addition to their cholesterol-lowering properties, simvastatin exerts a number of pleiotropic (non-lipid-lowering) vasculoprotective actions primarily responsible for its anti-ischemic and anti-anginal properties, so some systemic availability is desired $[3,4]$.

SV is a class II drug (low solubility, high permeability) according to the Biopharmaceutics classification system (BCS) [5]. Poor water solubility of SV combined with the extensive metabolism in the gut wall and liver (presystemic metabolism due to the affinity for CYP enzymes) results in a low systemic bioavailability (less than 5\%) when the drug is administered in a conventional dosage form (e.g. immediate-release (IR) tablets) [6,7]. These considerations also apply for other poorly-soluble CYP3A4-substrate drugs.

As one of the most promising formulation strategies, self-emulsifying drug delivery systems have been proposed to improve solubility and dissolution rate of SV [8-11]. The aforementioned approach is commonly used to resolve the issue of SV poor water solubility; however, none of the cited studies considered the extensive presystemic clearance of SV, which could in turn significantly decrease its systemic bioavailability if SV is delivered into the small intestine in a presolubilized form. 
Self-microemulsifying drug delivery systems (SMEDDS) are isotropic mixtures of drug, oil, surfactant, and cosurfactant, that under dilution in vivo can spontaneously form microemulsions with droplet size less than $50 \mathrm{~nm}$ [12]. These drug delivery systems allow a drug to remain in a dissolved state throughout its transit through the gastrointestinal (GI) tract, thus enhancing drug's permeation across the intestinal membrane [13]. Therefore, for poorly water soluble drugs that exhibit dissolution rate-limited absorption (such as SV), SMEDDS can enhance the rate and extent of absorption, and offer an advantage over conventional oral dosage forms [12]. It is assumed that various factors may be responsible for the enhanced absorption of SMEDDS-formulated drugs, including improved drug solubilization, and/or increased membrane permeability and lymphatic transport, although the exact mechanisms are not fully elucidated $[14,15]$. With a large variety of excipients, ranging from oils to surfactants and cosurfactants, there are many different combinations that could form SMEDDS [16]. The abilities of the non-ionic polyglycolyzed glycerides including PEG 300 oleic glycerides, PEG 400 caprylic/capric glycerides, propylene glycol monolaurate, and propylene glycol monocaprylate to formulate SMEDDS were widely investigated $[8,9,17]$. Due to their favourable properties, such as high solubilization capacity for poorly soluble drugs and low toxicity, these excipients have been increasingly used in SMEDDS [18]. However, when considering formulation of SV-loaded SMEDDS, one should have in mind that presystemic metabolism of this drug can significantly lower its bioavailability in case a solubilized drug is delivered as an IR formulation. Some data suggest that modified-release formulations of statins might increase drug hepatic availability by minimizing drug release in proximal parts of the GI tract, and favouring drug uptake from ileum and colon [3]. Therefore, it is assumed that SV-loaded SMEDDS filled in acid-resistant capsules can postpone the absorption of the drug, and consequently improve its systemic and hepatic bioavailability. 
Recent trends in pharmaceutical development highlight the benefits of in silico physiologically-based pharmacokinetic (PBPK) modeling as a useful tool to estimate drug absorption and/or bioavailability, and thus facilitate the selection of appropriate formulation strategy, and development of novel oral drug delivery systems $[19,20]$.

The aims of this study were (i) to develop and evaluate SMEDDS containing SV in order to explore their potential to improve drug solubility, and (ii) to predict the optimal place in the GI tract for the delivery of SV using coupled in vitro/in silico approach . In order to forecast pharmacokinetic (PK) behaviour of SV in humans, and provide mechanistic understanding of the processes the drug undergoes after oral administration of various types of oral SMEDDS formulations (immediate- and modified-release) PBPK modeling was used. In addition, the formulation approach applied in this study aimed to support the idea that the combined in vitro/in silico analysis should be embraced as an integral part of contemporary drug development.

\section{Materials and methods}

\section{Materials}

Simvastatin (Ph. Eur. grade) was obtained from Hemofarm a.d. (Serbia). PEG 400 caprylic/capric glycerides, propylene glycol monocaprylate, PEG 300 oleic glycerides, and propylene glycol monolaurate were obtained from Gattefossé (France). Polysorbate 80 was obtained from Sigma Aldrich Chemie GmbH (Germany). All other chemicals and reagents used were of analytical grade. 


\section{Methods}

\section{Solubility studies}

Solubility of SV in oils (PEG 300 oleic glycerides, propylene glycol monocaprylate, propylene glycol monolaurate), surfactant (PEG 400 caprylic/capric glycerides) and cosurfactant (polysorbate 80) was determined by adding an excess amount of the drug to a screw-capped glass vials containing $2 \mathrm{ml}$ of each excipient. The suspensions were shaken for $48 \mathrm{~h}$ at room temperature $\left(20 \pm 2{ }^{\circ} \mathrm{C}\right)$ using $\mathrm{IKA}^{\circledR} \mathrm{KS} 260 \mathrm{~B}$ shaker (IKA, Germany) at 350 rpm, and then centrifuged for $30 \mathrm{~min}$ at $3000 \mathrm{rpm}$ (MPW-56 centrifuge, MPW Med. Instruments, Poland). Aliquot of supernatant from each vial was diluted with ethanol $96 \%$ v/v, and drug content was quantified UV-spectrophotometrically (Evolution 300, Termo Fisher Scientific, England), since this method has been referenced in literature for quantification of SV [21,22]. Absorbance values were recorded at the wavelength of maximum absorption at $239 \mathrm{~nm}$, and drug concentrations was calculated from the relevant calibration curve $\left(r^{2} 0.999\right)$. Specificity, linearity, accuracy, precision and sensitivity of the applied method was confirmed by in house validation (data not shown).

Solubility studies were conducted in triplicate and the results are expressed as mean \pm standard deviation (S.D.) to illustrate the dispersion in the obtained data. The results were statistically analyzed with one-way analysis of variance (ANOVA), using the p-value limit of 0.05. In case of statistically significant differences within the obtained values $(p<0.05)$, post-hoc Tukey test was used to estimate the difference within each pair of values. The difference between the two values was considered significant if the calculated $Q$ value was larger than the critical $Q$ value of the studentized range distribution $(\alpha=0.05)$.. 


\section{Determination of microemulsion area}

Components of surfactant phase (surfactant $(\mathrm{S})$ and cosurfactant $(\mathrm{Cs})$ ) were mixed in three different S/Cs ratios: 1:1, 2:1 and 3:1 (w/w). SMEDDS were prepared when the selected oil was added to surfactant phase at nine different ratios $(9: 1,8: 2,7: 3,6: 4,5: 5,4: 6,3: 7,2: 8$ and 1:9, w/w). In order to observe the influence of drug on the microemulsion area, SV-loaded SMEDDS were prepared by adding the precise amount of SV $(5 \% \mathrm{w} / \mathrm{w})$ into the previously prepared SMEDDS and stirring on a magnetic stirrer (IKA ${ }^{\circledR}$ RH basic 2, IKA, Germany) at $50{ }^{\circ} \mathrm{C}$, until a clear solution was obtained. Each mixture was titrated with water in a dropwise manner while visually observed for transparency. During titration, samples were stirred on magnetic stirrer to allow equilibration. The "end point" was defined as the moment when clear mixtures turned turbid. Percentages of water, oil and surfactant phase, calculated at this point, were used to construct pseudoternary phase diagrams, and identify the microemulsion area in each diagram. The optimal formulations were selected at desired ratios of components based on the ability to form microemulsion.

\section{Droplet size determination}

The average droplet size and polydispersity index of unloaded and SV-loaded systems were determined immediately after dilution with purified water $(1: 10)$ by photon correlation spectroscopy (NanoZS90, Malvern Instruments, UK) at wavelength of $633 \mathrm{~nm}$ and a scattering angle of $90^{\circ}$. The results are presented as mean \pm S.D. of three consecutive measurements for each sample.

In order to evaluate the stability of the prepared samples average droplet size and polydispersity index were determined after three months, in the samples kept at room temperature $\left(20 \pm 2{ }^{\circ} \mathrm{C}\right)$. 


\section{Drug content determination}

SV content was determined in freshly prepared samples and after 3-months storage $(20 \pm 2$ ${ }^{\circ} \mathrm{C}$ ). Accurately weighted SMEDDS samples (equivalent to $20 \mathrm{mg}$ of SV) were dissolved in $10 \mathrm{ml}$ of ethanol 96\%, sonicated for $10 \mathrm{~min}$ on ultrasonic bath (Bandelin Sonorex Super RK $102 \mathrm{H}$, Bandelin electronic, Germany) and diluted with ethanol $96 \%$ to determine drug content at $239 \mathrm{~nm} \mathrm{UV}$-spectrophotometrically. Experiments were run in triplicate and the results are expressed as a percent of label claim (mean \pm S.D.).

\section{Dissolution studies}

The first set of experiments (setup I) comprised in vitro dissolution studies of SV-loaded SMEDDS (0.4 g of SMEDDS containing $20 \mathrm{mg} \mathrm{SV})$ filled in hard gelatin capsules size " 0 ". The selected experimental setup I followed the USP requirements for determination of simvastatin release rate from simvastatin tablets (USP 35), with an adjustment that excluded the addition $0.5 \%$ SDS in dissolution medium, in order to provide more discriminative test conditions. Dissolution tests were carried out in a rotating basket apparatus (Erweka DT70, Germany) at $75 \mathrm{rpm}$, and $37^{\circ} \mathrm{C}$ using $900 \mathrm{ml}$ phosphate buffer $\mathrm{pH} 7.0$ as dissolution medium. $5 \mathrm{ml}$ of samples were withdrawn at the predetermined time intervals $(5,10,15,30,45$, and 60 min), and replaced with equal volume of phosphate buffer. All samples were filtered, and analyzed for SV content UV-spectrophotometrically at the wavelength of maximum absorption (239 nm). Analytical procedure was adapted from Chavhan et al. [23]. Dissolution experiments were run in triplicate, and data were expressed as mean value \pm S.D.

The obtained dissolution profiles (setup I) were compared using model-independent mathematical approach that includes calculation of the similarity factor $\left(f_{2}\right)$ between two 
profiles [24]. Drug release profiles are considered similar if $f_{2}$ value ranges between 50 and $100[25]$.

In the second phase (setup II) $0.4 \mathrm{~g}$ the selected SV-loaded SMEDDS (containing $20 \mathrm{mg}$ of SV) was filled into hypromellose (HPMC)-based acid-resistant capsules size "0" (DRcaps ${ }^{\mathrm{TM}}$ capsules, Capsugel, USA) and assayed for drug release using reciprocating cylinder apparatus (Bio-Dis Extended Release Tester, VanKel Technology Group, USA) under the "the mediachange" conditions depicted in Table 1 . The proposed conditions were designed to simulate changes in physiological medium as the drug travels through GI tract under fasting conditions, because disintegration of acid-resistant capsules, and consequently drug release rate depend on media $\mathrm{pH}$. At the predetermined time intervals $(15,30,45,60,90,120,135$, $150,180,195$, and $210 \mathrm{~min}), 5 \mathrm{ml}$ of samples were collected and replaced with equal volume of dissolution medium. Determination of drug content followed the procedure applied in the setup I. The experiments were run in triplicate, and data were expressed as mean value \pm S.D.

\section{Physiologically-based simulation of simvastatin pharmacokinetics}

GastroPlus $^{\mathrm{TM}}$ software (version 9.0.0007, Simulations Plus, Inc., USA) was used to forecast SV pharmacokinetic properties following oral administration of different dosage forms. The software operates on the basis of the Advanced Compartmental Absorption and Transit (ACAT) of human GI tract that consists of nine compartments (stomach, duodenum, jejunum 1 and 2, ileum 1-3, caecum and ascendent colon), and uses a system of mathematical equations to simulate the effect of physiological conditions on drug transit, dissolution, absorption and metabolism in the GI tract [26].

The employed modeling approach consisted of two phases, whereas the first phase was model construction, followed by model exploration. The first phase referred to the selection of input data and evaluation of the simulation outcomes by comparison with the data from the in vivo 
study. Drug and formulation-related input parameters were experimentally determined, in silico estimated or taken from literature [Table 2]. Since the available human in vivo data referred to an IR tablet [2], drug dissolution rate under physiological conditions was predicted using Johnson dissolution model (based on modified Nernst-Bruner equation) [36]. All other ACAT model parameters were fixed at software default values that represent human fasted/fed physiology.

In order to simulate drug disposition and metabolism in different tissues, ACAT model was linked to the whole-body PBPK model. The selected PBPK model used GastroPlus ${ }^{\mathrm{TM}}$ default physiology parameters for 30 years $70 \mathrm{~kg}$ American (Western) human adult, based on population mean values obtained from published data. Drug-specific model was constructed using metabolite tracking feature to simultaneously describe conversion and pharmacokinetics of SV and SVA, as depicted in Figure 1.

Therefore, it accounted for the conversion of SV to SVA by intestinal and hepatic carboxylesterases (CES), and SV and SVA metabolism by CYP3A4 enzymes in the gut and liver. Michaelis constant $\left(\mathrm{K}_{\mathrm{m}}\right)$ and maximum reaction rate $\left(\mathrm{V}_{\max }\right)$ values for CES in the intestine and liver, and for CYP3A4 clearance of SV were taken from literature, as well as $\mathrm{K}_{\mathrm{m}}$ value for SVA metabolism by CYP3A4 [Table 2]. $\mathrm{V}_{\max }$ for CYP3A4 clearance of SVA was optimized to match the resultant SV and SVA pharmacokinetic profiles to the in vivo observed data [2]. CYP3A4 distribution factors for each gut compartment, and CYP3A4 expression in the liver were provided within the program built-in database for the selected physiology (values calculated based on reported data [37]), and CES expression in the liver was calculated from literature data [38], assuming the software default concentration of 38 mg microsomal protein/g liver. The drug was assumed to distribute freely across the membranes, meaning that the partition into the tissue is limited solely by the tissue blood flow rate. Thus all the tissues, except the liver, were treated as perfusion-limited. 
Tissue/plasma partition coefficients $\left(\mathrm{K}_{\mathrm{p}}\right)$ for perfusion-limited tissues were calculated using a modified Rodgers-Single (Lukacova) equation, where unbound fraction in tissue $\left(\mathrm{f}_{\mathrm{ut}}\right)$ was calculated using S+ equation [39]. In order to account for the OATP1B1-mediated transport of SVA across the basolateral hepatocytes membrane, the liver was set as a permeabilitylimited tissue, and SV and SVA partition into the tissue was optimized. OATP1B1 expression in the liver was estimated from literature data [40], as well as the kinetic parameter $\mathrm{K}_{\mathrm{m}}$ [41], and $\mathrm{V}_{\max }$ was a fitted value [Table 2]. In addition to active uptake, passive diffusion of unbound SVA across the basolateral membrane was simulated by using permeability-surface area product (PStc) for the liver, calculated on the basis of SVA passive diffusion clearance of $4 \mu \mathrm{l} / \mathrm{min} / 10^{6}$ cells [30]. On the contrary, the PStc value for SV across the hepatic basolateral membrane was assumed to be 10000 times greater than the hepatic blood flow, to reflect perfusion- and not permeability-limited entry and exit of lipophilic SV to and from the liver intracellular compartment [30].

The predictive power of the designed model was tested by comparing the simulation outcomes with digitized literature values on SV and SVA plasma concentration-time profiles following oral administration of a single $20 \mathrm{mg}$ dose IR SV tablet [2]. Digital extraction of published data from the graph was performed using DigIt ${ }^{\mathrm{TM}}$ program (version 1.0.4, Simulations Plus, Inc., USA). Since the participants in the reference study were given standard meals at 4 and $10 \mathrm{~h}$ after the drug administration, mixed multiple doses (MMD) option was used to switch from fasted to fed state, and accommodate food-induced changes in physiological parameters.

In the model exploration phase, in vitro obtained SV dissolution profiles were used as inputs to describe drug release rate in vivo and simulate the expected PK outcomes following oral administration of the selected experimental formulations. Furthermore, virtual dissolution profile served as input to estimate the influence of hypothetical changes in formulation 
parameters (i.e. variations in drug release rate) on the drug PK profile. All other input parameters were kept at the same values as determined in the model construction phase.

\section{Results}

\section{Characterization of SMEDDS}

Solubility of simvastatin

Experimentally obtained solubilities of SV in various oils, surfactant, and cosurfactant are

provided in Table 3. Solubility of SV in propylene glycol monocaprylate $(115.18 \pm 7.32$ $\mathrm{mg} / \mathrm{ml}$ ) was more than two times higher compared to drug's solubility in PEG 300 oleic glycerides $(53.34 \pm 8.89 \mathrm{mg} / \mathrm{ml})$. Overall, among the tested oils, SV exhibited the highest solubility in propylene glycol monocaprylate. The results of one-way ANOVA revealed statistically significant differences among the SV solubilities in different oils, since the calculated p-value was less than $0.05(\mathrm{p}$-value $=0.019)$. In addition, post-hoc Tukey test indicated statistically significant difference between the SV solubilities in PEG 300 oleic glycerides and propylene glycol monocaprylate.

\section{Determination of microemulsion area}

The pseudoternary phase diagrams were constructed for unloaded SMEDDS [Figures 2-4] and SV-loaded SMEDDS [Figures 5-7] to identify microemulsion regions, and optimize the concentration of oil, surfactant and cosurfactant in SMEDDS. The areas of transparent systems are depicted as grey sequences. For both, unloaded and SV-loaded systems, it was evident that three diagrams with propylene glycol monolaurate as oil phase [Figures 2 and 5] had smaller microemulsion areas in comparison to the diagrams with other two oils, propylene glycol monocaprylate [Figures 3 and 6], and PEG 300 oleic glycerides [Figures 4 
and 7]. Unloaded SMEDDS with propylene glycol monocaprylate as oil phase produced larger microemulsion area [Figure 3] compared to those with PEG 300 oleic glycerides [Figure 4]. Also, there was a slight difference among propylene glycol monocaprylate-based systems with different ratios of surfactant and cosurfactant, since the systems with 1:1 and 2:1 S/Cs ratios had slightly larger microemulsion area in contrast to systems with $3: 1 \mathrm{~S} / \mathrm{Cs}$ ratio [Figure 3].

It was observed that incorporation of $\mathrm{SV}(5 \% \mathrm{w} / \mathrm{w})$ led to a decrease in the microemulsion areas in all diagrams [Figures 5-7]. There was a noticeable decrease in the microemulsion areas for SV-loaded SMEDDS with propylene glycol monocaprylate as oil phase [Figure 6] compared to those systems without SV [Figure 3]. SV-loaded systems with propylene glycol monocaprylate had smaller microemulsion area in comparison to SV-loaded systems with PEG 300 oleic glycerides [Figure 7].

The results of this study demonstrated that only four mixtures gave clear systems, when titrated with water, and can be regarded as SMEDDS. These systems [marked as F1-F4; Table 4] were selected for further investigation.

\section{Droplet size analysis}

The average droplet size and polydispersity index of both unloaded (F1-F4) and SV-loaded (F1s-F4s) SMEDDS are presented in Table 5.

Unloaded SMEDDS (F1-F4), diluted immediately after preparation, had droplet size between $10.25 \mathrm{~nm}$ and $18.58 \mathrm{~nm}$. The average droplet size of SV-loaded SMEDDS, with PEG 300 oleic glycerides as oil phase (F1s-F3s), was between $13.57 \mathrm{~nm}$ and $17.45 \mathrm{~nm}$. However, it was observed that propylene glycol monocaprylate-based formulation (F4s) had larger droplet size $(46.41 \mathrm{~nm})$. 
As demonstrated in Table 5, the low values of polydispersity index (0.132 to 0.189$)$ indicated good uniformity of droplet size of the unloaded SMEDDS. Furthermore, the obtained results showed that polydispersity index of F1s-F3s formulations determined after preparation indicated greater uniformity of droplet size, in comparison to formulation F4s whose polydispersity index $>0.2$ implied larger variations of droplet size.

Droplet size and polydispersity index determined after 3-month storage of the samples at room temperature revealed small changes of these values for SV-loaded formulations F1sF3s, which implies that these systems are considered to be stable. Therefore, taking into account that F4s formulation initially had larger droplet size and polydispersity index and was prone to greater changes due to storage, only samples F1s-F3s were selected as promising drug delivery systems.

\section{Drug content determination}

The average value for SV content in freshly prepared SMEDDS samples was $100.13 \pm$ $0.42 \%$, which indicates that the applied preparation procedure and excipients did not affect the drug content. After 3-months storage, the determined drug content was $99.51 \pm 0.67 \%$ demonstrating that no notable changes happened during this period.

\section{In vitro dissolution data}

Figure 8 shows comparative in vitro dissolution profiles of $\mathrm{F} 1 \mathrm{~s}-\mathrm{F} 3 \mathrm{~s}$ formulations in hard gelatin capsules. In the first 5 min, more than $85 \% \mathrm{SV}$ was released from F2s and F3s formulations. However, SV release from F1s formulation was lower compared to F2s and F3s formulations, reaching approximately $80 \%$ within the same time. Since drug release from F1s was somewhat slower than from formulations F2s and F3s, formulation F1 was excluded from further studies. The selection of the optimal formulation for additional investigation 
under the experimental setup II was based on the properties of the excipients. Namely, formulation F3s contained slightly higher percent of PEG 400 caprylic/capric glycerides [Table 4], known for its high tolerance, low toxicity in animals, and the ability to enhance the intestinal absorption of drugs [42-44], and therefore this formulation F3s was chosen for additional testing.

The dissolution profile of the selected F3s formulation filled in acid-resistant capsules obtained under the setup II is shown in Figure 9. The acid-resistant capsules managed to sustain drug release in media $\mathrm{pH} 1.2$ and $\mathrm{pH}$ 6.0, whereas at $\mathrm{pH} 6.4$ the capsules started to disintegrate and gradually release the drug. Figure 9 also shows virtual dissolution profile (V1), generated to reflect the situation where $100 \%$ of the drug is dissolved in $180 \mathrm{~min}$, which was later used in the in silico study to assess the influence of variations in drug release rate on the drug PK profile.

\section{In silico predictions}

The model-building strategy applied in this study focused on simulation of the key processes influencing SV pharmacokinetics, while keeping the model as simple as possible. Drugspecific model was constructed based on the selected input parameters [Table 2], and population variability in the physiological and pharmacokinetic parameters was neglected, since the purpose of the in silico modeling was to estimate the influence of formulation factors on SV plasma exposure. Also, we need to note that the generated in silico results refer to a $70 \mathrm{~kg}$ human adult representative, but the designed model can be approximated to a wide range of body weights by adjusting the cardiac output and applying linear scaling to the tissue volumes and perfusion rates for the adjusted weight and cardiac output, respectively [45,46]. The predicted SV and SVA plasma concentration-time profiles are depicted in Figure 10, together with the corresponding values taken from literature [2]. Table 6 summarizes the 
predicted and in vivo observed SV/SVA PK parameters following oral administration of 20 mg SV IR tablets.

In order to assess the influence of formulation factors on the expected SV plasma profile, in vitro obtained dissolution profiles for $\mathrm{F} 3 \mathrm{~s}$ formulation filled in conventional hard gelatin capsules and acid-resistant capsules [Figures 11 and 12] were used as inputs in the designed SV-specific PBPK model. The simulated profiles are shown in Figure 11, and the generated PK parameters are depicted in Table 7. According to the obtained results, mere improvement in drug solubility, achieved by SV-loaded SMEDDS, will not affect SV plasma exposure $\left(0.97 \%\right.$ difference in area-under-the-curve $\left(\mathrm{AUC}_{0 \rightarrow \infty}\right)$ in comparison to the value simulated based on software calculated drug dissolution). On the other hand, simulations based on drug dissolution from SMEDDS filled in acid-resistant capsules indicated certain increase in SV bioavailability $\left(10.3 \%\right.$ and $10.2 \%$ increase in bioavailability and $\mathrm{AUC}_{0 \rightarrow \infty}$, respectively, in comparison to the values simulated based on software calculated drug dissolution) [Table 7].

\section{Discussion}

In contrast to $\mathrm{SV}$ poor water solubility $(0.026 \mathrm{mg} / \mathrm{ml}$ experimentally determined $)$, the drug displayed relatively high solubilities in the investigated excipients, which is in accordance with SV high $\log \mathrm{P}$ value of 4.71 [31]. As depicted in Table 3, the experimental values were within or close to the wide range of values found in literature $[8,9,11]$. It was assumed that propylene glycol monocaprylate has greater capacity to solubilize poorly water soluble drugs due to certain polarity of caprylic acid, as discussed in the previous study with atorvastatin [47].

The selection of the most appropriate components for SMEDDS should be based on the constructed pseudoternary diagrams. One of the crucial factors that influence the formulation 
of SMEDDS is hydrophilic-lipophilic balance (HLB) value of the surfactant. Surfactants with HLB value 12-15, such as PEG 400 caprylic/capric glycerides and polysorbate 80 used in this study, are considered to have good efficacy for self-emulsification [48]. As pointed out in a previous study [49], the size of microemulsion area is determined by physical and chemical properties of the oil, aqueous and surfactant phases. Low surface tension at the oil-water interface, the presence of highly fluid interfacial film of surfactant, and penetration and association of oil molecules with interfacial surfactant film are prerequisites for the formation of microemulsion [49]. The presence of propylene glycol monolaurate as oil phase made the emulsification process more difficult compared to propylene glycol monocaprylate and PEG 300 oleic glycerides, and consequently led to smaller microemulsion area in these diagrams. It has been reported that the drug incorporated in SMEDDS may have some effect on selfemulsification performance of a system [13], which was confirmed by the results of this study. Namely, incorporation of SV $(5 \% \mathrm{w} / \mathrm{w})$ into the investigated SMEDDS led to decrease in the microemulsion region. This phenomena might be attributed to the incorporation of the drug into the oil droplets, resulting in the swelling of the oil droplets and expansion of the lipid phase, which signifies that higher ratio of surfactant phase is required in order to form SMEDDS [17,50]. Therefore, based on these results, only four systems [Table 4] were chosen as potential SMEDDS.

Additional investigation of the formulated systems included droplet size analysis, which implied that all SV-loaded SMEDDS (F1s-F4s) formed microemulsions since the average droplet size was less than $50 \mathrm{~nm}$ [12]. However, drug loading had some impact on droplet size of propylene glycol monocaprylate-based formulation (F4s) in comparison to F1s-F3s formulations. These results revealed that the type of oil phase plays a vital role in the microemulsification process, as the sample prepared with propylene glycol monocaprylate as oil phase had significantly larger droplet size. 
After 3-month storage all unloaded SMEDDS showed similar results regarding droplet size and polydispersity index. In the case of SV-loaded SMEDDS, only PEG 300 oleic glyceridesbased formulations (F1s-F3s) could be regarded as stable after 3 months, which might be explained by the fact that PEG 300 oleic glycerides has higher HLB value (HLB=9) in comparison to propylene glycol monocaprylate (HLB=6), and good miscibility with PEG 400 caprylic/capric glycerides as surfactant [50].

The in vitro drug dissolution profiles from the selected formulations obtained under the experimental setup I were compared by calculating similarity factor. The calculated value $\mathrm{f}_{2}$ 53.55 for F2s and F3s formulations indicated that these dissolution profiles could be considered similar. However, comparison between F1s and F2s $\left(f_{2} 44.69\right)$ and between F1s and F3s $\left(f_{2} 36.65\right)$ showed that drug release profiles from these formulations were not similar, and that drug release from F1s was slower, which was one of the criteria to exclude this formulation from further studies (as indicated above).

Under the experimental setup II, the acid-resistant capsules stayed intact in acidic buffer $\mathrm{pH}$ 1.2, thus proving that the drug will not be released at low $\mathrm{pH}$ during the entire residence time in the stomach. The complete SV release occurred within 90 minutes in phosphate buffer 6.4. As already mentioned, design of SV oral dosage form with improved bioavailability requires a comprehensive analysis of formulation properties in conjunction with drug pharmacokinetic behaviour. SV undergoes a rather complex pharmacokinetics after oral administration, and the correct representation of the relevant PK properties in the in silico model is challenging. Several studies published in literature aimed to elucidate the underlying mechanisms that drive SV and SVA absorption, distribution and metabolism [30,31,51-53]. Nevertheless, substantial population variability in SV pharmacogenetics, namely polymorphisms in the genes encoding SV/SVA metabolizing enzymes and transporters [53-56], in addition to the variations in the reported values concerning drug-related biopharmaceutical properties, can 
be seen as a limitation for the accurate prediction of SV pharmacokinetics in a particular individual. Probably the most conceptual model that describes this drug pharmacokinetics is the recently published SV/SVA population model, developed by using an integrated population PBPK approach [30]. However, the complexity of this model, and the suggested modeling approach, might limit its wider application.

In this study, in silico estimated $4.66 \% \mathrm{SV}$ reaching the systemic circulation following intake of $20 \mathrm{mg}$ SV IR tablets [Table 7] agreed well with the reported drug bioavailability of less than $5 \%[6,7]$. The predicted maximum plasma concentration $\left(\mathrm{C}_{\max }\right)$ and $\mathrm{AUC}_{0 \rightarrow \infty}$ for $\mathrm{SV}$ and SVA [Table 6] fitted into the range of values from the reference study [2]. The largest deviation from the in vivo data was noted for the time to reach SVA peak plasma levels $\left(\mathrm{t}_{\max }\right)$, but this difference can be attributed to the large variability in the kinetics of SVA active uptake into the hepatocytes and concomitant metabolic clearance. Another annotation concerns a double peak phenomena in the mean plasma concentration profile observed in different in vivo studies [2,52,56]. Several hypothesis tried to explain these observations, including the influence of enterohepatic circulation, variable gastric emptying and intestinal transit time, site-specific absorption with different lag times and absorption rates, or irregular absorption process due to dynamically changing environment for drug dissolution in GI tract at each time of administration [2,52]. Although the designed model was not able to fully capture the second peak, simulation results using MMD option (visible "cusp" in the plasma profile corresponding to the meal time $4 \mathrm{~h}$ after drug administration [Figure 10]) indicate that intake of food might at least be partly responsible for the observed multiple peaks in SV plasma profiles.

Simulation results based on input drug dissolution profiles indicated improved SV bioavailability with greater delay in drug release [Table 7], and the applied modeling approach provided a mechanistic explanation of the obtained data. Namely, in the case of SV- 
loaded SMEDDS filled in hard gelatin capsules, small difference in $\mathrm{AUC}_{0 \rightarrow \infty}$ in comparison to the value simulated based on software calculated dissolution is expected because large portion of the drug, absorbed from the GI tract, will undergo first pass extraction in the gut and liver. This outcome signifies that application of different techniques with the sole aim to improve SV solubility might not be the best strategy in the formulation of SV oral dosage forms. However, in the case of acid-resistant capsules, there was an expected delay in drug release and absorption [Figures 11 and 12]. In this case, in silico results indicated reduced SV hydrolysis and first pass extraction by CYP3A4, which is predominantly distributed in the upper small intestine. Consequently, the simulations showed that increased fraction of SV dose reached the portal vein followed by, to a lesser extent, increase in systemic drug exposure [Table 7]. Predictions based on virtual dissolution profile, generated to reflect the situation where $100 \%$ of the drug is dissolved in 180 min [Figure 9, profile V1], imply that further delay in drug release would additionally increase systemic drug bioavailability, up to 7.20\% [Table 7]. Any further increase in plasma drug exposure would be limited by extensive hepatic drug metabolism. Overall, the obtained results suggest that formulation of an oral dosage form that releases the drug in a presolubilized form in distal parts of the intestine might improve SV bioavailability for more than $50 \%$ in comparison to a conventional IR dosage form.

\section{Conclusion}

In this study, self-microemulsifying drug delivery system was developed for simvastatin, whereas the selected SMEDDS formulation consisted of 10\% PEG 300 oleic glycerides, 67.5\% PEG 400 caprylic/capric glycerides and 22.5\% polysorbate 80 . Moreover, the outcomes of this study signify that application of different techniques with the sole aim to 
improve SV solubility is not the best strategy in the formulation of SV oral dosage forms because majority of the delivered dose would be cleared by presystemic metabolism in the small intestine. Instead, the proposed strategy should comprise solubility enhancement (e.g. SMEDDS formulation) and delayed drug release. Although the use of acid-resistant capsules was found helpful in protecting the drug against early degradation in GI tract, in silico modeling results indicated that $\mathrm{pH}$-controlled drug release system that dissolve above $\mathrm{pH} 7.0$ or colon delivery system might further improve SV bioavailability. The most important clinical benefit is that such an outcome is expected to enable SV dose reduction, and decrease the risk of SV-associated adverse effects. The proposed formulation strategy could also be applied to other poorly soluble CYP3A4-substrate drugs with similar absorption pattern. Further in vivo studies are encouraged to confirm our findings, and support applicability of the proposed in vitro/in silico approach to guide formulation strategy.

In addition, a simplified SV-specific PBPK model developed in this study provided a plausible explanation of SV/SVA pharmacokinetics, resulting in reasonable estimates of SV plasma exposure after oral administration. Therefore, it can potentially be used to assess the influence of formulation factors on drug absorption and disposition when developing SV oral dosage forms. However, more complex models that take into account population variability in physiological factors and SV pharmacogenetics are needed to enable predictions for different patients/population groups.

\section{Acknowledgements}

This work was done under the Project TR-34007 supported by the Ministry of Education, Science and Technological Development, Republic of Serbia. 


\section{Disclosure of interest}

The authors report no conflicts of interest.

\section{References}

[1] Schachter M. Chemical, pharmacokinetic and pharmacodynamic properties of statins: an update. Fundam Clin Pharmacol. 2005;19(1):117-125.

[2] Tubic-Grozdanis M, Hilfinger JM, Amidon GL, et al. Pharmacokinetics of the CYP3A substrate simvastatin following administration of delayed versus immediate release oral dosage forms. Pharm Res. 2008;25(2):1591-1600.

[3] Tiwari R, Pathak K. Statins therapy: a review on conventional and novel formulation approaches. J Pharm Pharmacol. 2011;63(8):983-998.

[4] Lardizabal JA, Deedwania PC. The anti-ischemic and anti-anginal properties of statins. Curr Atheroscler Rep. 2011;13(1):43-50.

[5] Kuentz M. Lipid-based formulations for oral delivery of lipophilic drugs. Drug Discov Today Technol. 2012;9(2):97-104.

[6] Thomas N, Holm R, Garmer M, et al. Supersaturated self-nanoemulsifying drug delivery systems (super-SNEDDS) enhance the bioavailability of the poorly water-soluble drug simvastatin in dogs. AAPS J. 2013;15(1):219-227.

[7] Mauro VF. Clinical pharmacokinetics and practical applications of simvastatin. Clin Pharmacokinet. 1993;24(3):195-202.

[8] Kang BK, Lee JS, Chon SK, et al. Development of self-microemulsifying drug delivery systems (SMEDDS) for oral bioavailability enhancement of simvastatin in beagle dogs. Int J Pharm. 2004;274(1-2):65-73. 
[9] Karim FT, Kalam A, Anwar R, et al. Preparation and evaluation of SEDDS of simvastatin by in vivo, in vitro and ex vivo technique. Drug Dev Ind Pharm. 2015;41(8):1338-1342.

[10] Thomas N, Müllertz A, Graf A, et al. Influence of lipid composition and drug load on the in vitro performance of self-nanoemulsifying drug delivery systems. J Pharm Sci. 2012;101(5):1721-1731.

[11] Mahmoud H, Al-Suwayeh S, Elkadi S. Design and optimization of self-nanoemulsifying drug delivery systems of simvastatin aiming dissolution enhancement. Afr J Pharm Pharmacol. 2013;7(22):1482-1500.

[12] Gursoy RN, Benita S. Self-emulsifying drug delivery systems (SEDDS) for improved oral delivery of lipophilic drugs. Biomed Pharmacother. 2004;58(3):173-182.

[13] Pouton CW. Lipid formulations for oral administration of drugs: non-emulsifying, selfemulsifying and 'self-microemulsifying' drug delivery systems. Eur J Pharm Sci. 2000 Oct;11 Suppl 2:93-98.

[14] O'Driscoll CM, Griffin BT. Biopharmaceutical challenges associated with drugs with low aqueous solubility-the potential impact of lipid-based formulations. Adv Drug Deliv Rev. 2008;60(6):617-624.

[15] Dahan A, Hoffman A. Rationalizing the selection of oral lipid based drug delivery systems by an in vitro dynamic lipolysis model for improved oral bioavailability of poorly water soluble drugs. J Control Release. 2008;129(1):1-10.

[16] Kohli K, Chopra S, Dhar D, et al. Self-emulsifying drug delivery systems: an approach to enhance oral bioavailability. Drug Discov Today. 2010;15(21-22):958-965.

[17] Qureshi MJ, Mallikarjuna C, Kian WG. Enhancement of solubility and therapeutic potential of poorly soluble lovastatin by SMEDDS formulation adsorbed on directly compressed spray dried magnesium aluminometasilicate liquid loadable tablets: a study in diet induced hyperlipidemic rabbits. Asian J Pharm Sci. 2010;10(1):40-56. 
[18] Strickley RG. Solubilizing excipients in oral and injectable formulations. Pharm Res. 2004;21(2):201-230.

[19] Jones HM, Chen Y, Gibson C, et al. Physiologically based pharmacokinetic modeling in drug discovery and development: a pharmaceutical industry perspective. Clin Pharmacol Ther. 2015;97(3):247-262.

[20] Kostewicz ES, Aarons L, Bergstrand M, et al. PBPK models for the prediction of in vivo performance of oral dosage forms. Eur J Pharm Sci. 2014;57:300-321.

[21] More AS, Firake BM, Firke SD. Spectrophotometric methods for estimation of simvastatin in bulk drug and its dosage form. Anal Chem Ind J. 2016;16(6):258-264.

[22] Kalarani DH, Venkatesh P, Reddy KR et al. Simple and precise method for the estimation of simvastatin in formulations by UV-visible spectrophotometer. Res J Pharm Technol. 2009;2(4):878-879.

[23] Chavhan V, Reddy K, Ahhirao K. Development of UV spectrophotometric methods and validation for estimation of simvastatin in bulk and tablet dosage form by absorbance maxima and area under the curve method. J App Pharm. 2014;6(1):55-64.

[24] Moore JW, Flanner HH. Mathematical comparison of dissolution profiles. Pharm Technol. 1996;20:64-74.

[25] Food and Drug Administration/ Center for Drug Evaluation and Research [Internet]. Guidance for Industry: dissolution testing of immediate release solid oral dosage forms; 1997 [cited 23 December 2016]. Available from: http://www.fda.gov/downloads/drugs//guidances/ucm070237.pdf

[26] Agoram B, Woltosz WS, Bolger MB. Predicting the impact of physiological and biochemical processes on oral drug bioavailability. Adv Drug Deliv Rev. 2001 Oct;50 Suppl $1: 41-67$. 
[27] Ishigami M, Honda T, Takasaki W, et al. A comparison of the effects of 3-hydroxy-3methylglutaryl-coenzyme a (HMG-CoA) reductase inhibitors on the CYP3A4-dependent oxidation of mexazolam in vitro. Drug Metab Dispos. 2001;29(3):282-288.

[28] Pasanen MK, Neuvonen M, Neuvonen PJ, et al. SLCO1B1 polymorphism markedly affects the pharmacokinetics of simvastatin acid. Pharmacogenet Genomics. 2006;16(12):873-879.

[29] Hochman JH, Pudvah N, Qiu J, et al. Interactions of human P-glycoprotein with simvastatin, simvastatin acid, and atorvastatin. Pharm Res. 2004;21(9):1686-1691.

[30] Tsamandouras N, Dickinson G, Guo Y, et al. Development and application of a mechanistic pharmacokinetic model for simvastatin and its active metabolite simvastatin acid using an integrated population PBPK approach. Pharm Res. 2015;32(6):1864-1883.

[31] Gertz M, Houston JB, Galetin A. Physiologically based pharmacokinetic modeling of intestinal first-pass metabolism of CYP3A substrates with high intestinal extraction. Drug Metab Dispos. 2011;39(9):1633-1642.

[32] Imai N, Ohtawa M. Ring-opening hydrolases for simvastatin in plasma, liver and intestinal microsomes of rats. Drug Metab Pharmacokinet. 1992;7(5):599-608.

[33] Ishigam $\mathrm{M}$, Uchiyama $\mathrm{M}$, Kondo $\mathrm{T}$, et al. Inhibition of in vitro metabolism of simvastatin by itraconazole in humans and prediction of in vivo drug-drug interactions. Pharm Res. 2001;18(5):622-631.

[34] Prueksaritanont T, Ma B, Yu N. The human hepatic metabolism of simvastatin hydroxy acid is mediated primarily by CYP3A, and not CYP2D6. $\mathrm{Br} \mathrm{J}$ Clin Pharmacol. 2003;56(1):120-124.

[35] Gelissena IC, Andrew J. McLachlan AJ. The pharmacogenomics of statins. Pharm Res. 2014;88:99-106. 
[36] Lu AT, Frisella ME, Johnson KC. Dissolution modeling: factors affecting the dissolution rates of polydisperse powders. Pharm Res. 1993;10(9):1308-1314.

[37] Paine MF, Khalighi M, Fisher JM, et al. Characterization of interintestinal and intraintestinal variations in human CYP3A-dependent metabolism. J Pharmacol Exp Ther. 1997;283(3):1552-1562.

[38] Ross MK, Borazjani A, Wang R, et al. Examination of the carboxylesterase phenotype in human liver. Arch Biochem Biophys. 2012; 522(1):44-56.

[39] Lukacova V, Parrott N, Lave T, et al. General approach to calculation of tissue:plasma partition coefficients for physiologically based pharmacokinetic (PBPK) modeling. AAPS National Meeting; 2008 Nov 15-20; Atlanta, GA.

[40] Badée J, Achour B, Rostami-Hodjegan A, et al. Meta-analysis of expression of hepatic organic anion-transporting polypeptide (OATP) transporters in cellular systems relative to human liver tissue. Drug Metab Dispos. 2015;43(4):424-432.

[41] Huang H. Characterization of in vitro systems for transporter studies [master's thesis]. Uppsala (Sweden): Biology Education Centre and Department of Pharmacy, Uppsala University; 2010.

[42] Hu Z, Tawa R, Konishi T, et al. A novel emulsifier, Labrasol, enhances gastrointestinal absorption of gentamicin. Life Sci. 2001;69(24):2899-2910.

[43] Prasad YV, Puthli SP, Eaimtrakarn S, et al. Enhanced intestinal absorption of vancomycin with Labrasol and D-alpha-tocopheryl PEG 1000 succinate in rats. Int J Pharm. 2003;250(1):181-190.

[44] Koga K, Kusawake Y, Ito Y, et al. Enhancing mechanism of Labrasol on intestinal membrane permeability of the hydrophilic drug gentamicin sulfate. Eur J Pharm Biopharm. 2006;64(1):82-91. 
[45] Samant TS, Lukacova V, Schmidt S. Development and qualification of physiologically based pharmacokinetic models for drugs with atypical distribution behavior: A desipramine case study. CPT Pharmacometrics Syst Pharmacol. 2017;6(5):315-321.

[46] Li GF, Wang K, Chen R, et al. Simulation of the pharmacokinetics of bisoprolol in healthy adults and patients with impaired renal function using whole-body physiologically based pharmacokinetic modeling. Acta Pharmacol Sin. 2012;33(11):1359-71.

[47] Bandivadekar M, Pancholi S, Kaul-Ghanekar R, et al. Single non-ionic surfactant based self-nanoemulsifying drug delivery systems: formulation, characterization, cytotoxicity and permeability enhancement study. Drug Dev Ind Pharm. 2013;39(5):696-703.

[48] Thi TD, Van Speybroeck M, Barillaro V, et al. Formulate-ability of ten compounds with different physicochemical profiles in SMEDDS. Eur J Pharm Sci. 2009;38(5):479-488.

[49] El Maghraby GM. Transdermal delivery of hydrocortisone from eucalyptus oil microemulsion: effects of cosurfactants. Int J Pharm. 2008;355(1-2):285-292.

[50] Balakrishnan P, Lee BJ, Oh DH, et al. Enhanced oral bioavailability of coenzyme Q10 by self-emulsifying drug delivery systems. Int J Pharm. 2009;374(1-2):66-72.

[51] Lippert J, Brosch M, von Kampen O, et al. A mechanistic, model-based approach to safety assessment in clinical development. CPT Pharmacometrics Syst Pharmacol. 2012;1:e13.

[52] Jin SJ, Bae KS, Cho SH, et al. Population pharmacokinetic analysis of simvastatin and its active metabolite with the characterization of atypical complex absorption kinetics. Pharm Res. 2014;31(7):1801-1812.

[53] Tsamandouras N, Dickinson G, Guo Y, et al. Identification of the effect of multiple polymorphisms on the pharmacokinetics of simvastatin and simvastatin acid using a population-modeling approach. Clin Pharmacol Ther. 2014;96(1):90-100. 
[54] Birmingham BK, Bujac SR, Elsby R, et al. Impact of ABCG2 and SLCO1B1 polymorphisms on pharmacokinetics of rosuvastatin, atorvastatin and simvastatin acid in Caucasian and Asian subjects: a class effect? Eur J Clin Pharmacol. 2015;71(3):341-355.

[55] Ramsey LB, Johnson SG, Caudle KE, et al. The clinical pharmacogenetics implementation consortium guideline for SLCO1B1 and simvastatin-induced myopathy: 2014 update. Clin Pharmacol Ther. 2014;96(4):423-428.

[56] Cheng H, Rogers JD, Sweany AE, et al. Influence of age and gender on the plasma profiles of 3-hydroxy-3-methylglutaryl-coenzyme A (HMG-CoA) reductase inhibitory activity following multiple doses of lovastatin and simvastatin. Pharm Res. 1992;9(12):16291633. 
Figure 1

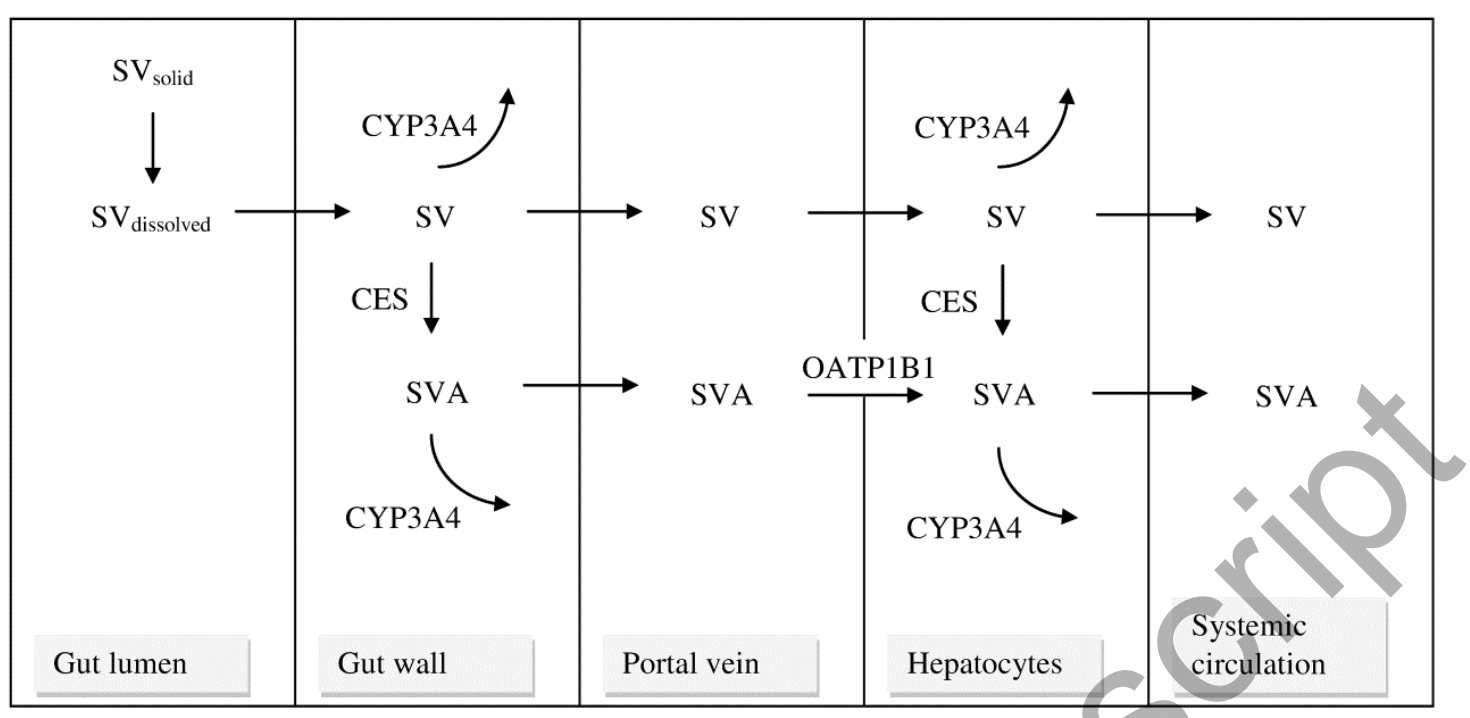

Table 1. Dissolution test conditions for the experimental setup II 

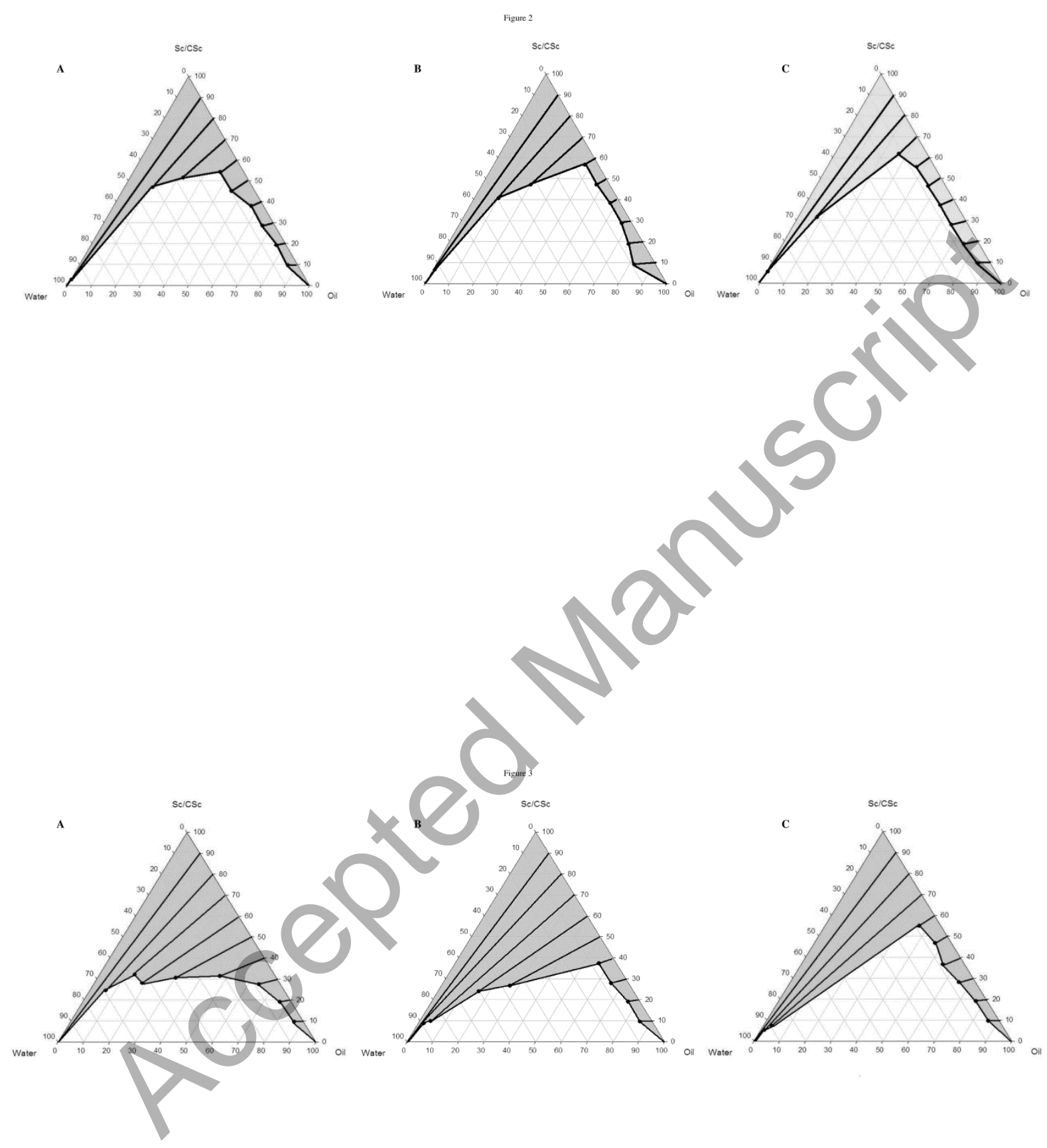

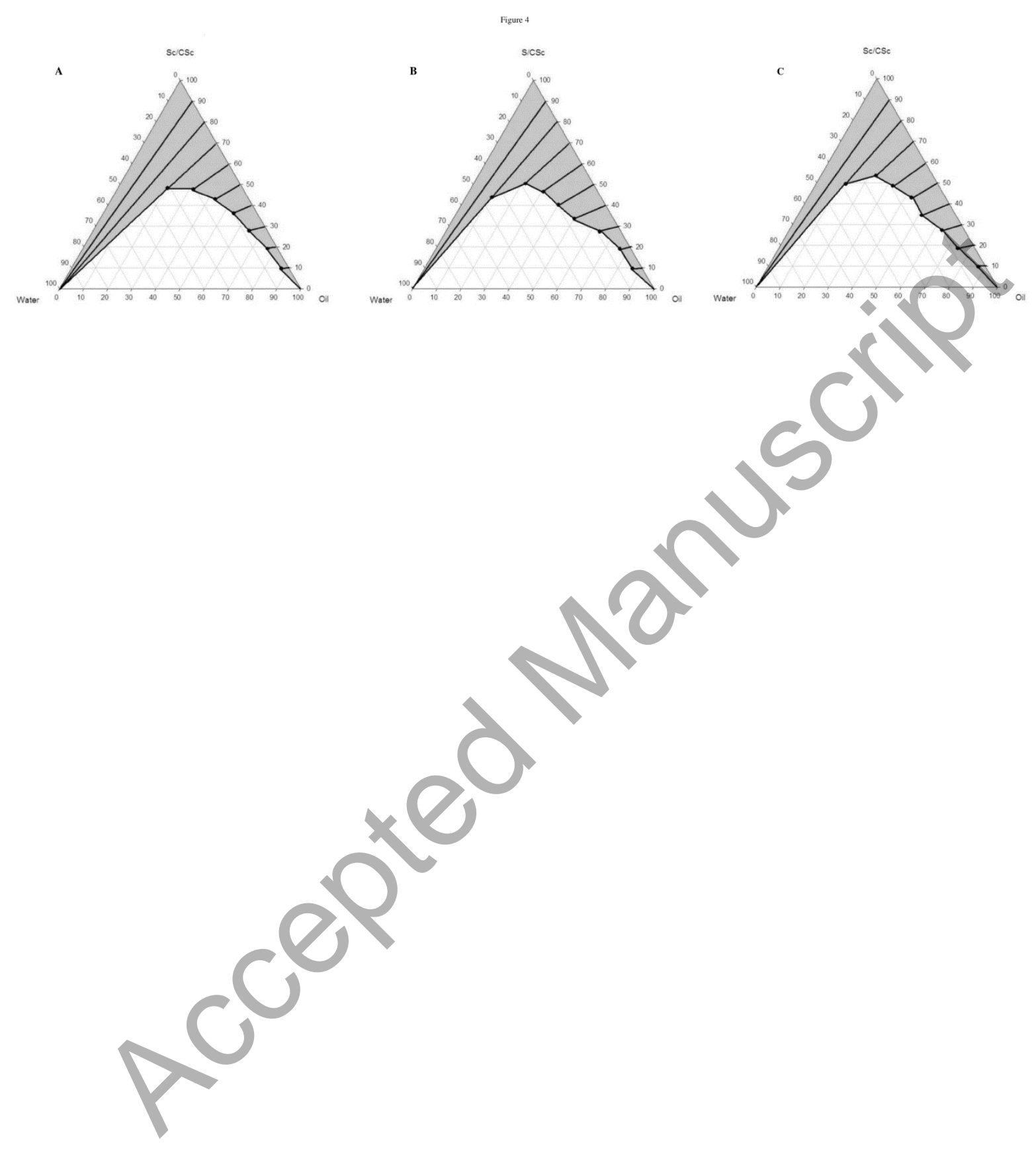

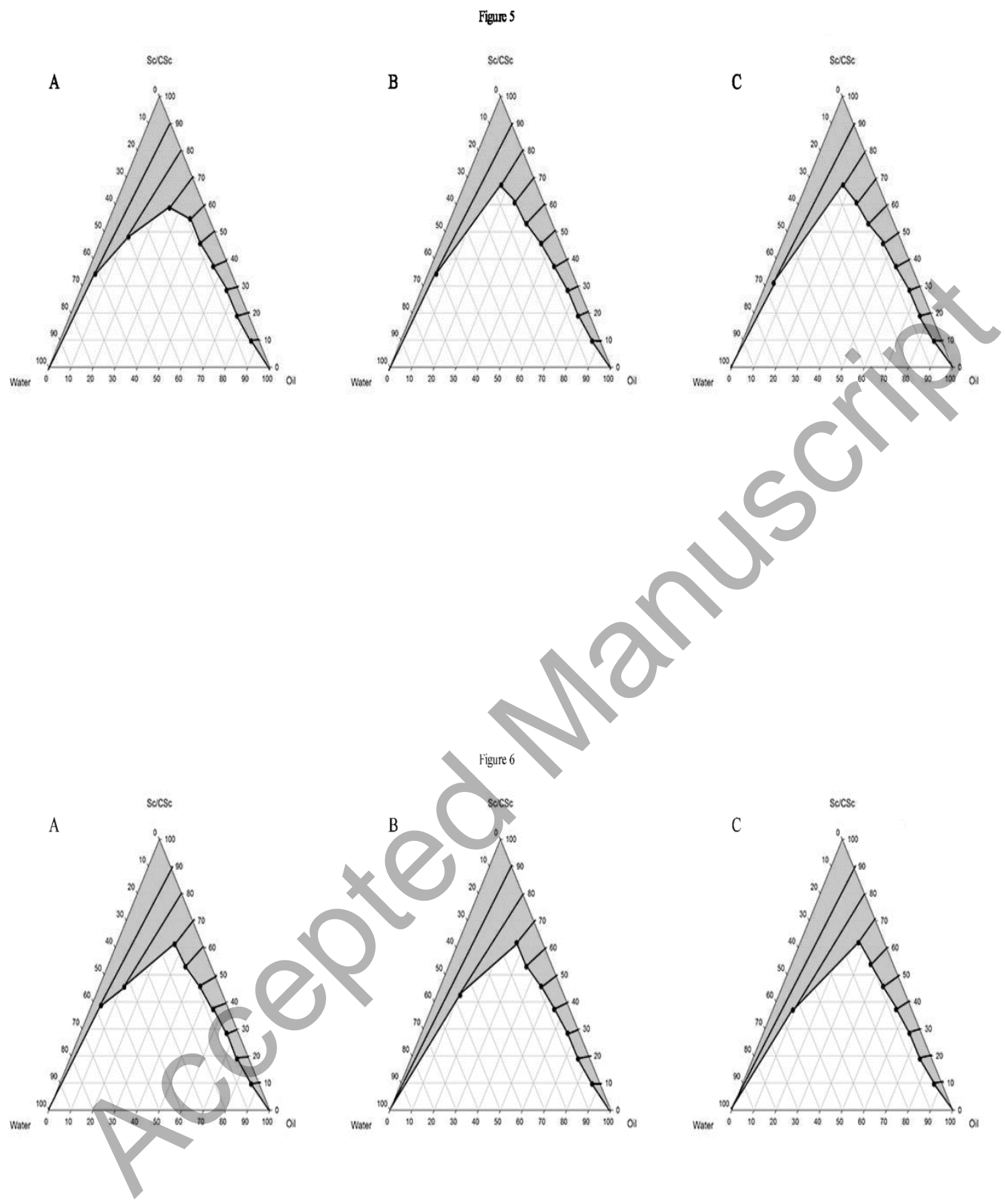

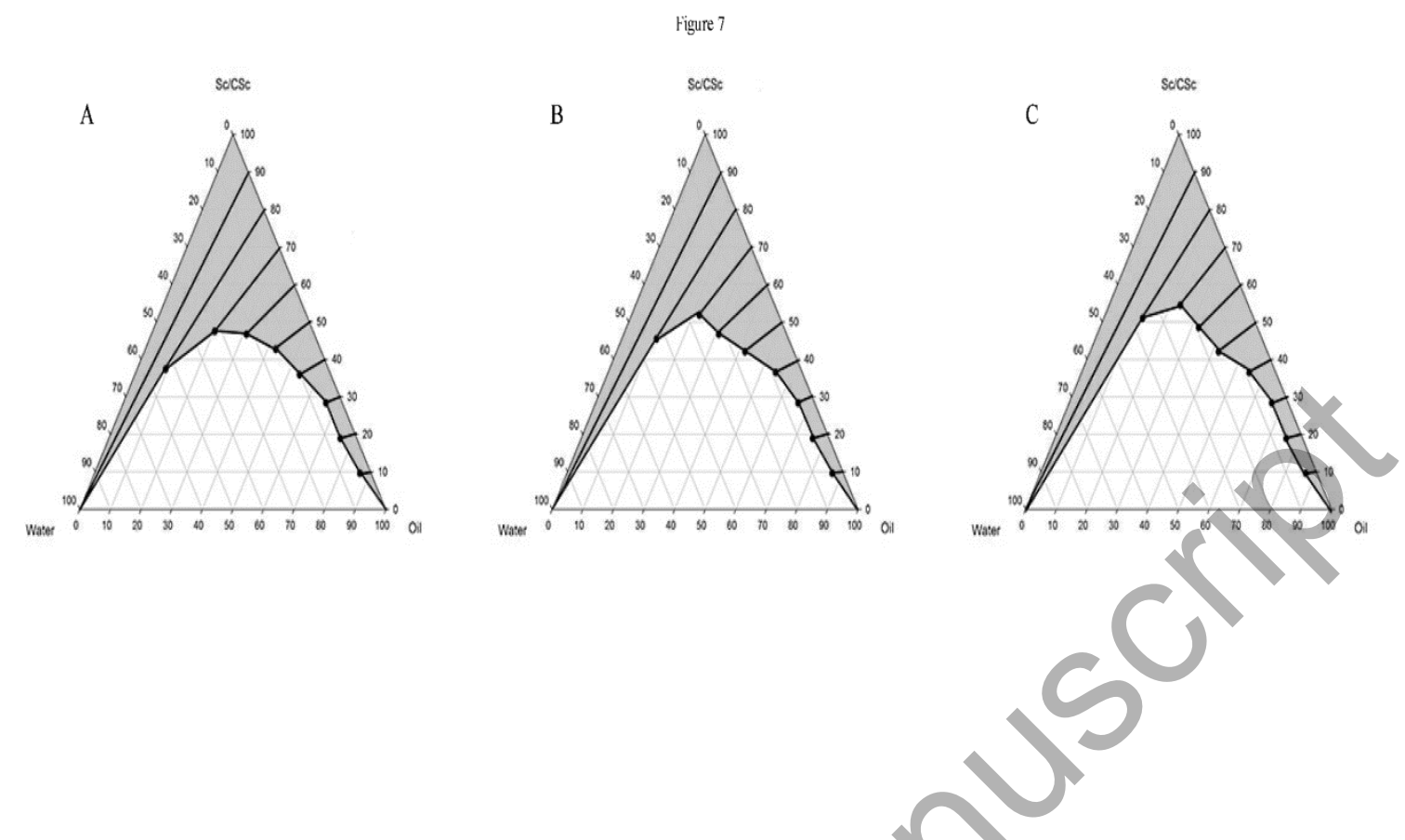


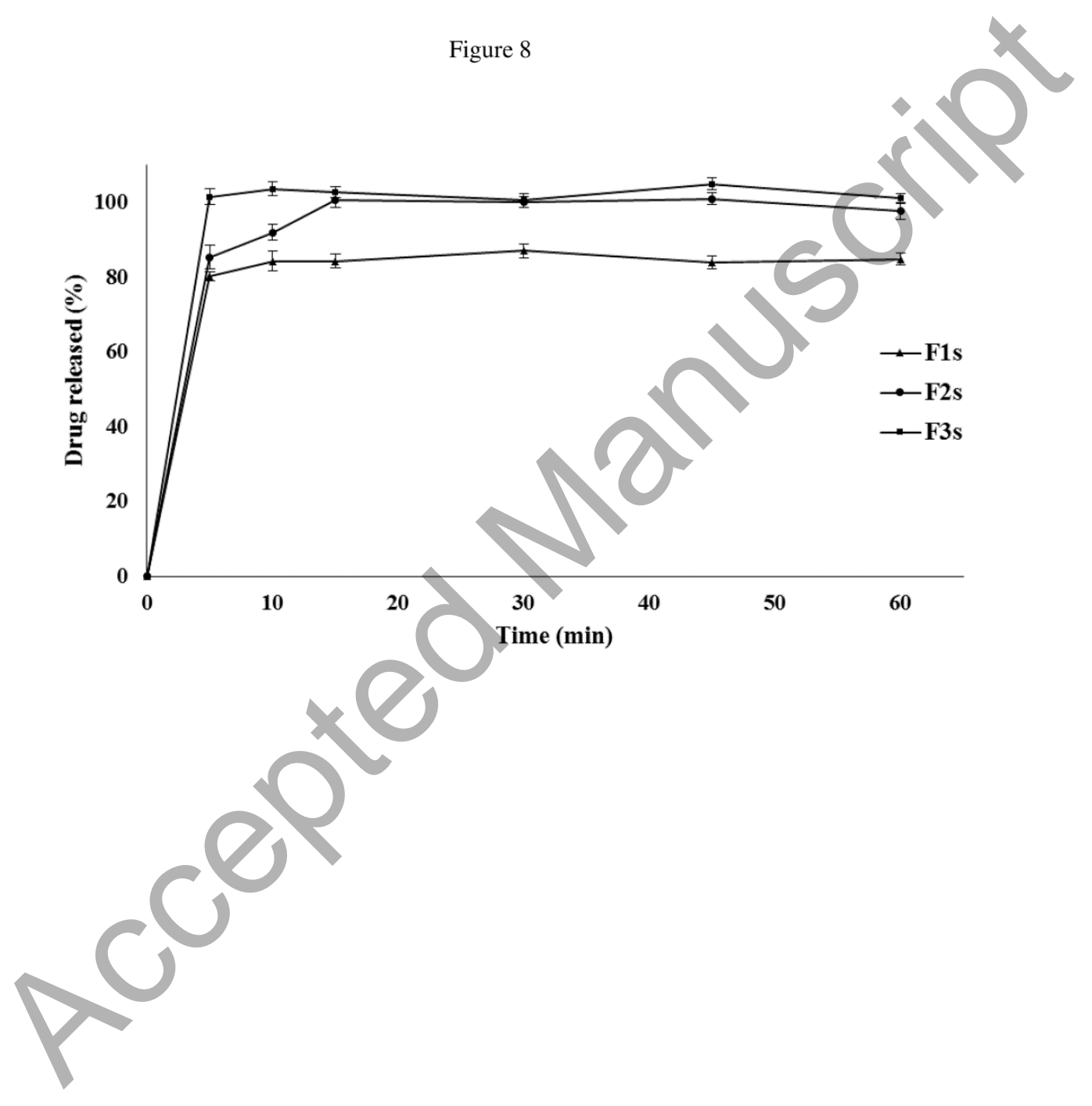




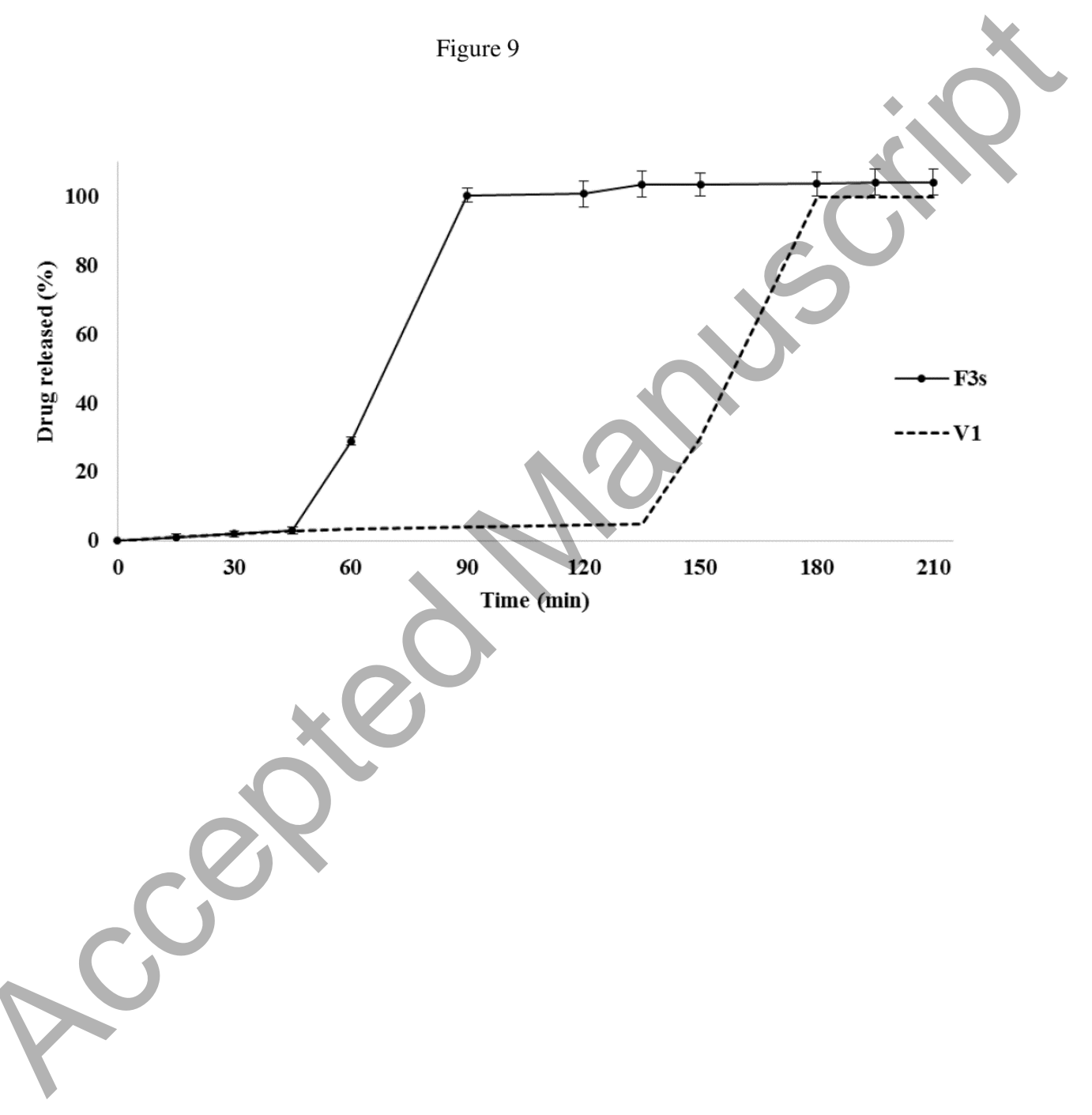


Figure 10

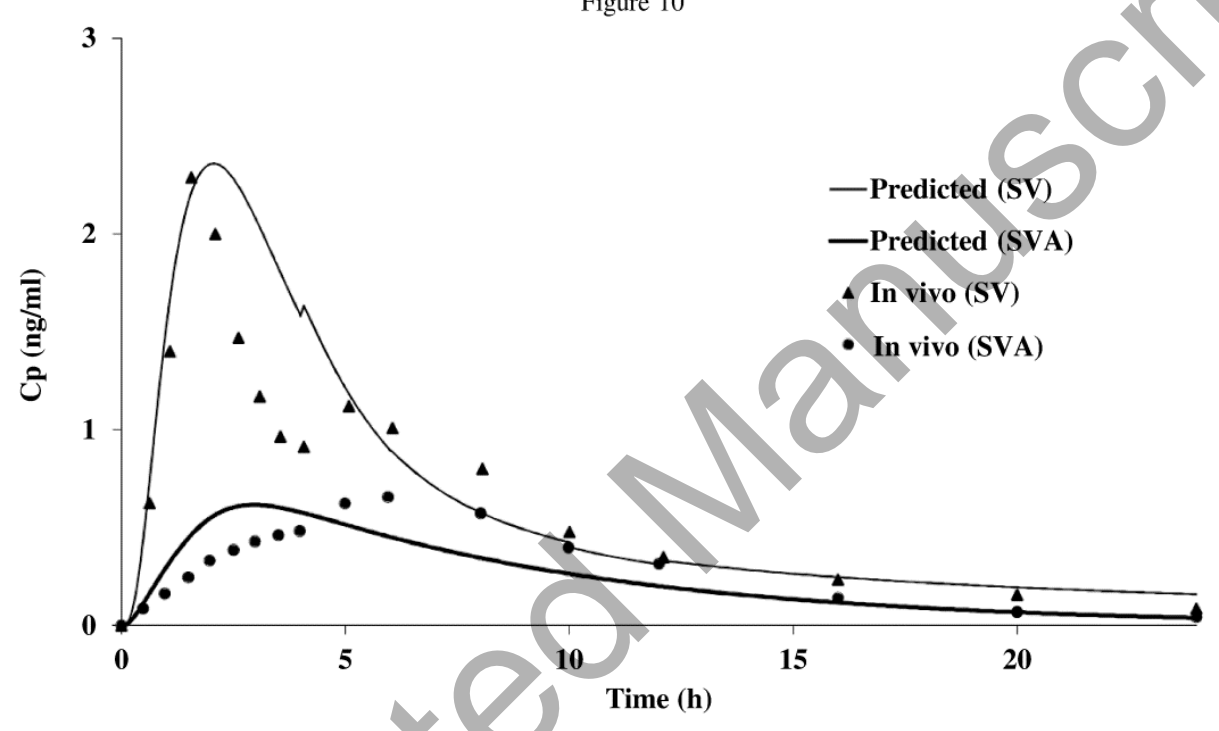




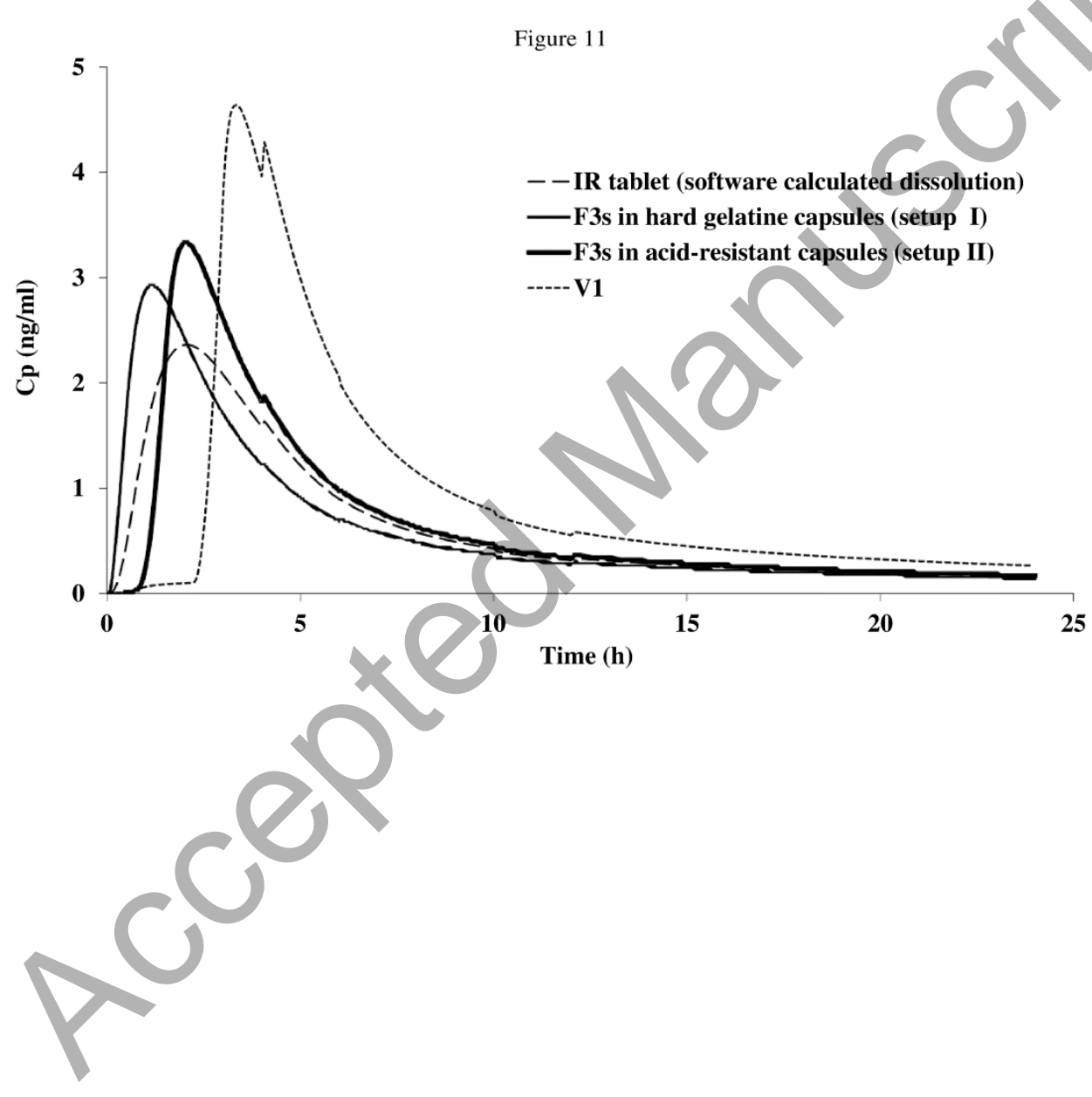




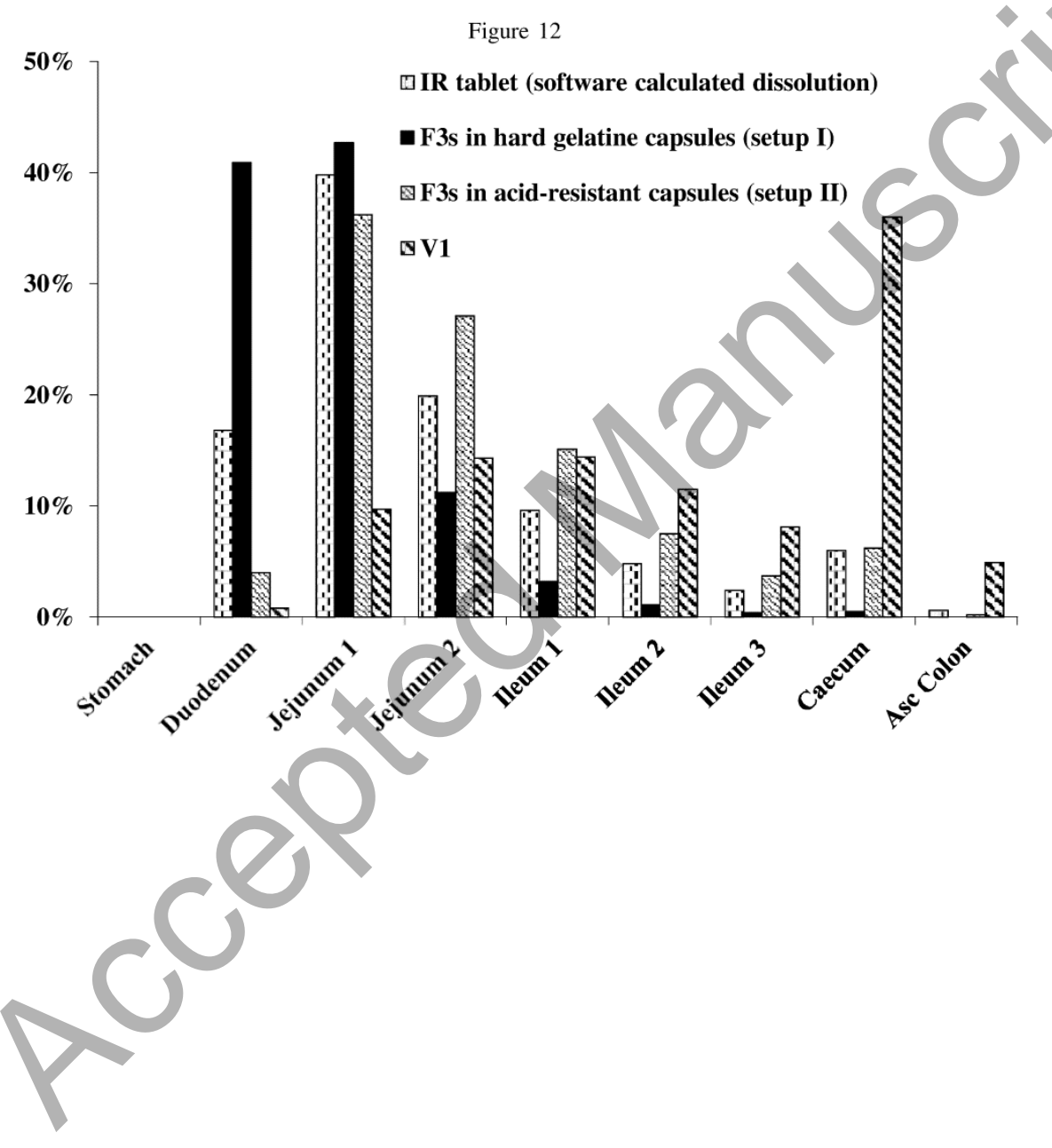


Table 1. Dissolution test conditions for the experimental setup II

\begin{tabular}{llllll}
\hline Row & I & II & III & IV & V \\
\hline Represented GI region & stomach & duodenum & proximal & distal & distal \\
& & & jejunum & jejunum & ileum \\
pH value & $\mathrm{pH} 1.2$ & $\mathrm{pH} 6.0$ & $\mathrm{pH} \mathrm{6.4}$ & $\mathrm{pH} \mathrm{6.9}$ & $\mathrm{pH} \mathrm{7.4}$ \\
Residence time (min) & 15 & 15 & 90 & 60 & 30 \\
Hold dip time (s) & 30 & & & & \\
Drain time (s) & 60 & & & & \\
\hline
\end{tabular}

Table 2. Summary of simvastatin/simvastatin acid PBPK model input parameters

\begin{tabular}{|c|c|c|}
\hline Parameter & Value (SV) & Value (SVA) \\
\hline Molecular weight & $418.60 \mathrm{~g} / \mathrm{mol}$ & $436.60 \mathrm{~g} / \mathrm{mol}$ \\
\hline $\log \mathrm{D}(\mathrm{pH} 7.0)$ & $4.40^{\mathrm{a}}$ & \\
\hline $\mathrm{pK}_{\mathrm{a}}$ & $13.49^{\mathrm{b}}$ & \\
\hline Human effective permeability, $\mathrm{P}_{\text {eff }}$ & $3.20 \times 10^{-4} \mathrm{~cm} / \mathrm{s}^{\mathrm{c}}$ & - \\
\hline Drug dose & & - \\
\hline Dose volume & & - \\
\hline Solubility (aqueous) & $20 \mu \mathrm{g} / \mathrm{ml}^{\mathrm{e}}$ & - \\
\hline Mean precipitation time & $900 \mathrm{~s}^{\mathrm{f}}$ & - \\
\hline Diffusion coefficient & $6.42 \times 10^{-6} \mathrm{~cm}^{2} / \mathrm{s}^{\mathrm{g}}$ & - \\
\hline Drug particle density & $1.20 \mathrm{~g} / \mathrm{ml}^{\mathrm{f}}$ & - \\
\hline Effective particle radius & $9 \mu \mathrm{m}^{\mathrm{h}}$ & - \\
\hline Blood/plasma concentration ratio & $0.57^{\mathrm{i}}$ & $0.56^{\mathrm{h}}$ \\
\hline Unbound percent in plasma & $1.34 \%^{\mathrm{h}}$ & $5.48 \%^{\mathrm{h}}$ \\
\hline Intestinal CES $\mathrm{K}_{\mathrm{m}}$ & $237.60 \mathrm{mg} / \mathrm{l}^{\mathrm{j}}$ & - \\
\hline
\end{tabular}




\begin{tabular}{lll}
\hline Intestinal CES V & $0.09 \mathrm{mg} / \mathrm{s}^{\mathrm{j}}$ & - \\
Hepatic CES K & & - \\
Hepatic CES V & $85.10 \mathrm{mg} / \mathrm{l}^{\mathrm{j}}$ & - \\
CYP3A4 K & $0.40 \mathrm{mg} / \mathrm{s}^{\mathrm{j}}$ & $11.06 \mathrm{mg} / \mathrm{l}^{\mathrm{l}}$ \\
CYP3A4 V $_{\max }$ & $2.01 \mathrm{mg} / \mathrm{l}^{\mathrm{k}}$ & $800 \mathrm{mg} / \mathrm{s}^{\mathrm{m}}$ \\
OATP1B1 K & $0.57 \mathrm{mg} / \mathrm{s}^{\mathrm{k}}$ & $1.10 \mathrm{mg} / \mathrm{l}^{\mathrm{n}}$ \\
OATP1B1 V & - & $1 \times 10^{-5} \mathrm{mg} / \mathrm{s} / \mathrm{mg}$ transporter \\
\hline
\end{tabular}

${ }^{\mathrm{a}}$ Taken from [27]

${ }^{\mathrm{b}}$ Taken from [28]

${ }^{\mathrm{c}}$ Calculated from LLC-PK1 cell lines passive permeability [29]; software integrated permeability converter

d Taken from [2]

${ }^{\mathrm{e}}$ Experimental value

${ }^{\mathrm{f}}$ GastroPlus ${ }^{\mathrm{TM}}$ default

${ }^{\mathrm{g}}$ GastroPlus ${ }^{\mathrm{TM}}$ predicted

$\mathrm{h}$ Taken from [30]

${ }^{\mathrm{i}}$ Taken from [31]

${ }^{\mathrm{j}}$ Converted from in vitro obtained values [32]; software integrated unit converter

${ }^{\mathrm{k}}$ Converted from in vitro obtained values [33]; software integrated unit converter

${ }^{1}$ Converted from in vitro obtained value [34]; software integrated unit converter

${ }^{\mathrm{m}}$ Optimized values

${ }^{\mathrm{n}}$ Converted from in vitro obtained value [35]; software integrated unit converter 
Table 3. Solubility of simvastatin in various excipients

\begin{tabular}{|c|c|c|}
\hline Excipient & $\begin{array}{l}\text { Experimental values } \\
(\mathrm{mg} / \mathrm{ml})\end{array}$ & $\begin{array}{l}\text { Range of literature values } \\
(\mathrm{mg} / \mathrm{ml})\end{array}$ \\
\hline PEG 300 oleic glycerides & $53.34 \pm 8.89 *$ & $\begin{array}{l}30.00-40.96 \\
\text { [from ref. 8,9] }\end{array}$ \\
\hline Propylene glycol monocaprylate & $115.18 \pm 7.32$ & $\begin{array}{l}105.00-165.66 \\
\text { [from ref. } 9,11]\end{array}$ \\
\hline Propylene glycol monolaurate & $89.88 \pm 12.51$ & $\begin{array}{l}22.00-111.63 \\
{[\text { from ref. } 9,11]}\end{array}$ \\
\hline PEG 400 caprylic/capric glycerides & $90.64 \pm 13.42$ & $\begin{array}{l}76.45-78.00 \\
\text { [from ref. 8,9] }\end{array}$ \\
\hline Polysorbate 80 & $73.49 \pm 6.02$ & $\begin{array}{l}53.68-117.00 \\
\text { from ref. } 8.91\end{array}$ \\
\hline
\end{tabular}

* mean \pm S.D. 
Table 4. Composition of the selected SMEDDS

\begin{tabular}{lllll}
\hline Components $(\% \mathrm{w} / \mathrm{w})$ & $\mathrm{F} 1$ & $\mathrm{~F} 2$ & $\mathrm{~F} 3$ & $\mathrm{~F} 4$ \\
\hline PEG 300 oleic glycerides & 10.0 & 10.0 & 10.0 & $/$ \\
Propylene glycol & $/$ & $/$ & $/$ & 10.0 \\
monocaprylate & & & & \\
PEG 400 caprylic/capric & 45.0 & 60.0 & 67.5 & 67.5 \\
glycerides & & & & \\
Polysorbate 80 & 45.0 & 30.0 & 22.5 & 22.5 \\
\hline
\end{tabular}


Table 5. The average droplet size and polydispersity index of unloaded and simvastatinloaded SMEDDS

\begin{tabular}{lllll}
\hline \multicolumn{4}{l}{ Average droplet size $(\mathrm{nm})$} & \multicolumn{2}{l}{ Polydispersity index } \\
\hline after preparation & after 3 months & after preparation & after 3 months \\
\hline F1 & $10.25 \pm 0.07^{*}$ & $10.98 \pm 0.06$ & $0.132 \pm 0.007$ & $0.144 \pm 0.003$ \\
F3 & $11.90 \pm 0.14$ & $12.11 \pm 0.09$ & $0.189 \pm 0.006$ & $0.121 \pm 0.005$ \\
F4 & $11.68 \pm 0.12$ & $13.73 \pm 0.04$ & $0.137 \pm 0.016$ & $0.130 \pm 0.005$ \\
F1s & $18.58 \pm 0.04$ & $20.88 \pm 0.09$ & $0.155 \pm 0.005$ & $0.162 \pm 0.007$ \\
F2s & $13.57 \pm 0.06$ & $12.30 \pm 0.06$ & $0.181 \pm 0.006$ & $0.147 \pm 0.006$ \\
F3s & $16.03 \pm 0.03$ & $16.30 \pm 0.06$ & $0.183 \pm 0.010$ & $0.160 \pm 0.010$ \\
F4s & $46.41 \pm 0.17$ & $29.71 \pm 0.21$ & $0.286 \pm 0.001$ & $0.182 \pm 0.011$ \\
\hline
\end{tabular}

* mean \pm S.D. 
Table 6. Comparison of the predicted and observed simvastatin/simvastatin acid pharmacokinetic parameters for $20 \mathrm{mg}$ simvastatin oral dose

\begin{tabular}{lll}
\hline Parameter & Predicted & Observed* [from ref. 2] \\
\hline $\mathrm{SV} \mathrm{C}_{\max }(\mathrm{ng} / \mathrm{ml})$ & 2.36 & $2.68 \pm 1.33$ \\
$\mathrm{SV} \mathrm{t}_{\max }(\mathrm{h})$ & 2.08 & $2.4 \pm 1.65$ \\
$\mathrm{SV} \mathrm{AUC}_{0 \rightarrow \infty}(\mathrm{ng} \mathrm{h} / \mathrm{ml})$ & 18.62 & $14.81 \pm 7.28$ \\
$\mathrm{SVA} \mathrm{C}_{\max }(\mathrm{ng} / \mathrm{ml})$ & 0.62 & $0.73 \pm 0.36$ \\
$\mathrm{SVA} \mathrm{t}_{\max }(\mathrm{h})$ & 2.96 & $6.1 \pm 1.34$ \\
$\mathrm{SVA} \mathrm{AUC}_{0 \rightarrow \infty}(\mathrm{ng} \mathrm{h} / \mathrm{ml})$ & 6.19 & $7.27 \pm 3.40$ \\
\hline * mean \pm S.D. & &
\end{tabular}

* mean \pm S.D. 
Table 7. Predicted simvastatin pharmacokinetic parameters for different formulations

\begin{tabular}{lllll}
\hline Parameter & Based on & Based on & Based on & Based on \\
& software & dissolution data & dissolution data & virtual \\
& calculated drug & for F3s filled in & for F3s filled in & dissolution \\
& dissolution rate & hard gelatin & acid-resistant & profile (V1) \\
& & capsules & capsules & \\
& & (setup I) & (setup II) & \\
\hline $\mathrm{F}_{\mathrm{a}}{ }^{\mathrm{a}}(\%)$ & 100.00 & 99.99 & 99.82 \\
$\mathrm{FD}_{\mathrm{p}}{ }^{\mathrm{b}}(\%)$ & 19.26 & 21.40 & 28.53 \\
$\mathrm{~F}_{\mathrm{b}}{ }^{\mathrm{c}}(\%)$ & 4.66 & 4.56 & 5.14 & 7.20 \\
$\mathrm{C}_{\mathrm{max}}(\mathrm{ng} / \mathrm{ml})$ & 2.36 & 2.94 & 3.34 & 4.64 \\
$\mathrm{t}_{\max }(\mathrm{h})$ & 2.08 & 1.20 & 2.08 & 3.36 \\
$\mathrm{AUC}_{0 \rightarrow \infty}(\mathrm{ng} \mathrm{h} / \mathrm{ml})$ & 18.62 & 18.44 & 20.52 & 27.95 \\
\hline
\end{tabular}

${ }^{\mathrm{a}} \mathrm{F}_{\mathrm{a}}$ - Percent of drug dose absorbed (entered the enterocytes)

${ }^{\mathrm{b}} \mathrm{FD}_{\mathrm{p}}$ - Percent of drug dose reaching the portal vein

${ }^{c} F_{b}$ - Drug bioavailability (as a percent of dose) 


\section{Figure captions:}

Figure 1. Model scheme of simvastatin and simvastatin acid pharmacokinetics

Figure 2. Pseudoternary phase diagrams of the systems with propylene glycol monolaurate as oil phase and PEG 400 caprylic/capric glycerides/polysorbate 80 in ratios: (A) 1:1, (B) 2:1, and (C) 3:1

Figure 3. Pseudoternary phase diagrams of the systems with propylene glycol monocaprylate as oil phase and PEG 400 caprylic/capric glycerides/polysorbate 80 in ratios: (A) 1:1, (B) 2:1, and (C) 3:1

Figure 4. Pseudoternary phase diagrams of the systems with PEG 300 oleic glycerides as oil phase and PEG 400 caprylic/capric glycerides/polysorbate 80 in ratios:(A) 1:1, (B) 2:1, and (C) 3:1

Figure 5. Pseudoternary phase diagrams of simvastatin-loaded systems with propylene glycol monolaurate as oil phase and PEG 400 caprylic/capric glycerides/polysorbate 80 in ratios: (A) $1: 1$, (B) 2:1, and (C) 3:1

Figure 6. Pseudoternary phase diagrams of simvastatin-loaded systems with propylene glycol monocaprylate as oil phase and PEG 400 caprylic/capric glycerides/polysorbate 80 in ratios: (A) $1: 1,(B) 2: 1$, and (C) $3: 1$

Figure 7. Pseudoternary phase diagrams of simvastatin-loaded systems with PEG 300 oleic glycerides as oil phase and PEG 400 caprylic/capric glycerides/polysorbate 80 in ratios: (A) $1: 1$, (B) $2: 1$, and (C) $3: 1$

Figure 8. Comparative dissolution profiles of simvastatin-loaded SMEDDS obtained in a basket apparatus (setup I) 
Figure 9. Simvastatin dissolution profile from F3s formulation in a reciprocating cylinder apparatus (setup II); V1 refer to the virtual dissolution profile illustrating 100\% drug release in $3 \mathrm{~h}$

Figure 10. Predicted and in vivo observed [from ref. 2] simvastatin and simvastatin acid plasma concentration-time profiles following oral administration of $20 \mathrm{mg}$ simvastatin immediate-release tablet

Figure 11. Predicted simvastatin plasma concentration-time profiles for F3s formulation filled in conventional and acid-resistant hard gelatine capsules, along with the profile predicted based on virtual dissolution V1

Figure 12. Predicted compartmental absorption of simvastatin following oral administration of different formulations 